\title{
VARIABILIDADE ESPACIAL E DISPONIBILIDADE DE COBRE E ZINCO EM SOLOS DE VINHEDOS E ADJACÊNCIAS ( $\left.{ }^{1}\right)$
}

\author{
GUSTAVO SOUZA VALLADARES $\left(\left(^{2 *}\right)\right.$; EMÍLIO CARLOS DE AZEVEDO $\left({ }^{3}\right)$; \\ OTÁVIO ANTÔNIO DE CAMARGO $\left({ }^{4}\right)$; CÉLIA REGINA GREGO $\left(^{5}\right)$; \\ ALESSANDRA MARIA CIA SILVA RASTOLDO $\left({ }^{6}\right)$
}

\begin{abstract}
RESUMO
Com o tempo, o uso agrícola pode levar à contaminação do solo por metais pesados, principalmente por se servir de insumos e agroquímicos que possuem cobre e zinco em sua composição. O objetivo deste trabalho foi determinar os teores de cobre e zinco disponíveis no solo extraídos com DTPA e avaliar sua distribuição espacial empregando a geoestatística em áreas de vinhedos comerciais e experimentais e sob outros usos e coberturas nas adjacências das plantações. Foram coletadas amostras de terra nas profundidades de 0,0 a $0,15 \mathrm{~m}$ e 0,15 a $0,30 \mathrm{~m}$, em cem pontos amostrais. A área em estudo refere-se a duas microbacias localizadas no Centro de Frutas em Jundiaí (SP), sendo representativa da região vitícola, no relevo de mares de morros, com solos classificados predominantemente como Cambissolos e Argissolos. Os dados foram analisados utilizando estatística descritiva, teste de hipótese para médias e análises de correlação linear simples de Pearson. Para a análise espacial foram utilizados semivariogramas, semivariograma cruzado e a krigagem para a interpolação de dados e geração de mapas de isolinhas. Foram verificados altos teores de cobre e zinco nos solos da área de estudo e, ainda, maiores nos solos sob vinhedos, possivelmente em virtude da aplicação de agroquímicos. Nos solos sob vinhedos, os teores de cobre possuem correlação positiva com os teores de zinco, pH, matéria orgânica e saturação por bases. Os teores de cobre e zinco foram maiores nos vinhedos comerciais em comparação com os experimentais. A análise espacial dos dados permitiu avaliar a distribuição espacial dos teores de cobre e zinco, possibilitando, também, a verificação da correlação espacial positiva entre esses elementos na camada subsuperficial de 0,15 a 0,30m, com base no semivariograma cruzado.
\end{abstract}

Palavras-chave: krigagem, fertilidade do solo, DTPA, viticultura, metal pesado.

\section{ABSTRACT \\ SPATIAL VARIABILITY AND COPPER AND ZINC AVAILABILITY IN VINEYARDS AND NEARBY SOILS.}

With time, the intensive agricultural use of soil may lead to its contamination with heavy metals, mainly under the intense use of agrochemicals that are composed by zinc and copper. The objective of this work was to assess the concentration of available copper and zinc, extracted by DTPA, and evaluate its spatial distribution using geostatistics in experimental and commercial vineyards and under other land uses and covers in the surroundings of the cultivated areas. Soil samples were collected at the 0.0-

( $\left.{ }^{1}\right)$ Recebido para publicação em 15 de agosto de 2007 e aceito em 11 de fevereiro de 2009.

$\left(^{2}\right)$ Universidade Federal do Ceará/CCA/Departamento de Ciências do Solo, Campus do Pici, bloco 807, 60021-970 Fortaleza (CE). E-mail: valladares@ufc.br $\left(^{*}\right)$ Autor correspondente.

(3) Universidade Federal de Mato Grosso/FAMEV, Av. Fernando Correa da Costa s/n, Boa Esperança, 78060-900 Cuiabá (MT). E-mail: emilioaz@ufmt.br

$\left({ }^{4}\right)$ Instituto Agronômico (IAC), Centro de Solos e Recursos Agroambientais, Av. Barão de Itapura, 1481, Guanabara, 13020-902 Campinas (SP). E-mail: ocamargo@iac.sp.gov.br. Pesquisador do CNPq.

$\left(^{5}\right)$ Embrapa Monitoramento por Satélite, Av. Soldado Passarinho, 303, Chapadão, 13070-175 Campinas (SP). E-mail: crgrego@cnpm.embrapa.br

$\left.{ }^{(6}\right)$ PUC-Campinas, Rua Esmeralda Ferreira Oliveira, 96, Parque da Floresta, 13172-752 Sumaré (SP) E-mail: alessandra-cia@uol.com.br 
0.15 and $0.15-0.30 \mathrm{~m}$ depth in 100 locations. Two catchment areas located at the APTA-Fruits Center of the Secretary of Agriculture of São Paulo State in Jundiaí, Brazil were used in that study. It is a vineyard area with predominance of Umbrept and Udult soils. Initially data were assessed with descriptive statistics, hypothesis test and Pearson linear simple correlation. For the spatial analysis it was utilized semivariograms and crossed semivariograms, and kriging for data interpolation and isolines map generation. High contents of copper and zinc were verified in the area, being the higher ones in the soil under vineyard, probably due to the intensive use of agrochemicals. In these latter soils it was found high positive correlation between copper and zinc content, $\mathrm{pH}$, organic matter, and base saturation. Higher copper and zinc contents were found in the commercial vineyards compared to the experimental fields. The spatial analysis of data allowed to evaluate the spatial distribution of copper and zinc content as well as to verify the positive spatial correlation between those two elements in the $0.15-0.30 \mathrm{~m}$ depth by crossed semivariogram.

Key words: kriging, soil fertility, DTPA, vine, heavy metal.

\section{INTRODUÇÃO}

A qualidade do solo é fundamental ao desenvolvimento sustentável e à preservação dos ecossistemas e da biodiversidade. Nesse contexto, a contaminação do solo por metais pesados põe em risco sua capacidade produtiva e o equilíbrio dos ecossistemas. O solo é o ambiente de interface entre a rocha, o ar e a água e, conseqüentemente, está sujeito à contaminação por diferentes atividades antrópicas como, por exemplo, a agricultura, podendo ser fonte de poluição para seres vivos, sedimentos e corpos de água (FACCHINELLI et al., 2001).

Os solos naturalmente possuem metais pesados em concentrações variadas, dependendo do material de origem sobre o qual se formou, dos processos de formação e da composição e proporção dos componentes da sua fase sólida (FADIGAS, 2002; CAMARGO et al., 2003). As atividades antrópicas podem afetar a concentração de metais pesados no solo.

A água de irrigação, a aplicação de fertilizantes e o uso de agrotóxicos, assim como esgotos e dejetos de origem industrial e residencial podem contribuir para sua contaminação com metais pesados (FAcCHINelli et al., 2001; COSTA, 2002; Nicholson et al., 2003). A classe de solo, o relevo, a geologia e os processos erosivos influenciam a concentração e distribuição dos metais pesados no ambiente, afetando inclusive sua biodisponibilidade (RAmAlHo et al., 2000; Costa, 2002).

Com o tempo, o uso agrícola pode levar à contaminação do solo por metais pesados, de modo específico, do cobre nas áreas sob vinhedo (DelUISA et al., 1996; Giovannini, 1997; BRUn et al., 1998; Facchinelli et al., 2001; Parat et al., 2002; Alleoni et al., 2003; Chaignon e Hinsinger; 2003; ChAignon et al., 2003, Felix, 2005). O uso de insumos e agroquímicos que além do cobre possuem o zinco em sua composição, pode contaminar o solo por esses dois elementos (PAOlEtti et al, 1998; Ramos e LóPez-AcEvedo, 2004; GAw et al., 2006; RAMOS, 2006).
Os efeitos dessa contaminação refletem-se na própria agricultura, incluindo fitotoxicidade por altas concentrações, na manutenção dos processos microbiológicos e na transferência de elementos em níveis tóxicos ao homem e aos animais (NICHOLSON et al., 2003).

Os Sistemas de Informações Geográficas (SIG) são conjuntos manuais ou computacionais de procedimentos desenvolvidos para trabalhar com dados referenciados por coordenadas geográficas ou espaciais (STAR e Estes, 1990). Consistem em sistemas de suporte à decisão, integrando dados referenciados espacialmente em um ambiente de respostas a problemas (CÂmara e Medeiros, 1996; Ortiz et al., 2007) e que podem ser trabalhados com ferramentas da geoestatística (VIEIRA, 1997).

A intensificação do uso dessas técnicas na ciência do solo decorre do fato de se assumir que a distribuição espacial de pontos de observação tenha correlação, ou seja, que exista dependência espacial. Esta pressuposição não é verdadeira para a estatística clássica, na qual os pontos de observação são independentes, o que, na maioria dos casos, não ocorre nos estudos envolvendo as ciências da terra (Webster, 1985; Srivastava, 1996). Assim, segundo Couto e Klamt (1999), em estudos de solos, é comum presumir-se que determinados atributos possam ser representados pela média e variância, a partir de um número suficientemente grande de amostras. SRIVASTAVA (1996) comenta que a geoestatística incorpora, além da análise da distribuição estatística dos dados coletados, também as relações espaciais entre estes, na forma de correlação entre os pontos amostrados. De acordo com Trangmar et al. (1985), amostras mais próximas, dentro de uma mesma mancha de solo, são mais parecidas do que as mais distantes. Por essas diferenças, segundo Goovaerts (1997), os problemas da ciência da terra são efetivamente analisados atualmente por técnicas da geoestatística, quando a interpretação da distribuição espacial dos dados tem forte impacto sobre os resultados e a tomada de decisão. 
Desta forma, na região de Piemont, Itália, o uso de geoestatística e SIG foram eficientes nas determinações das relações entre os metais pesados e variáveis como a geologia e o uso e cobertura das terras, observando que os teores de cobre e zinco nas áreas sob vinhedos geravam fortes anomalias positivas (FAcchinelli et al., 2001). CARvalho et al. (2003) observaram dependência espacial de atributos físicos e químicos em solo sob vinhedos no Estado de São Paulo.

O objetivo deste trabalho foi determinar os teores de cobre e zinco disponíveis no solo, extraídos com DTPA, e avaliar suas distribuições espaciais empregando a geoestatística em áreas de vinhedos comerciais e experimentais e sob outros usos e coberturas nas adjacências das plantações.

\section{MATERIAL E MÉTODOS}

O trabalho foi desenvolvido em área experimental do Instituto Agronômico (IAC), e em área comercial adjacente, no município de Jundiaí (SP). A área de estudo pertence a duas microbacias hidrográficas, com aproximadamente 50 ha, com variado uso do solo, como videiras recentes e outras com mais de três décadas de idade, vegetação nativa, plantios florestais e pastagem nas adjacências dos vinhedos (Figura 1). Esta área localiza-se entre 680 e
$760 \mathrm{~m}$ de altitude, com geomorfologia de morros e relevo ondulado e forte ondulado. O clima corresponde ao Cwa de Köppen. Quanto à geologia, predominam os xistos. Os solos são classificados Como: Cambissolo Háplico Tb Distrófico, Latossolo Amarelo Distrófico, Argissolo Amarelo Distrófico e Gleissolo Háplico Tb Distrófico.

Os solos com vinhedos da região de estudo normalmente recebem calcário dolomítico em quantidade necessária para atingir $80 \%$ da saturação por bases e adubações anuais de $\mathrm{P}_{2} \mathrm{O}_{5}$ entre 100 e 200 $\mathrm{kg} \mathrm{ha}^{-1}$ de $\mathrm{N}$ entre 100 e $200 \mathrm{~kg} \mathrm{ha}^{-1}$ e de $\mathrm{K}_{2} \mathrm{O}$ entre 60 e $150 \mathrm{~kg} \mathrm{ha}^{-1}$, dependendo do produtor. Quanto ao manejo fitossanitário, são realizadas entre 2 e 4 aplicações com fungicidas contendo cobre ou zinco, podendo ser calda bordaleza ou outros agroquímicos como o mancozeb.

Foram coletados 100 pontos em duas profundidades $(0,0$ a $0,15 \mathrm{~m}$ e 0,15 a $0,30 \mathrm{~m})$, sendo 27 pontos sob vinhedos e 73 em solos com outros usos nas mesmas profundidades, totalizando 200 amostras. Todos os pontos amostrais foram georreferenciados com auxílio de GPS (Global Position System, modelo Garmin eTrex). Os teores de cobre e zinco foram determinados seguindo metodologia de RAIJ et al. (2001) pelo método do DTPA.

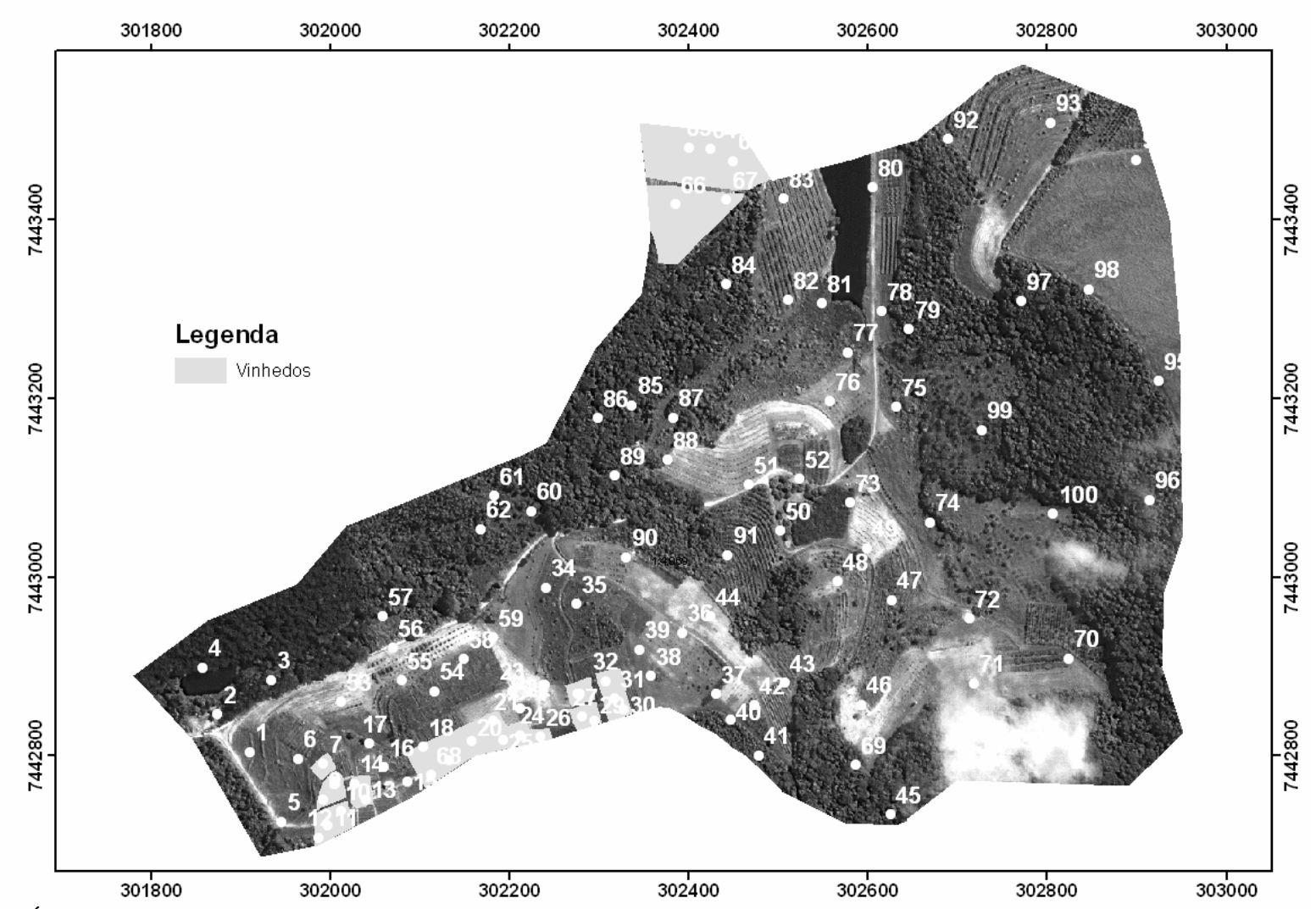

Figura 1. Área de estudo no Centro de Frutas em Jundiaí (SP), com a localização dos pontos amostrais de solos. 
Os dados foram analisados, para exploração inicial, por meio da estatística descritiva pelo programa STAT segundo VIEIRA et al. (2002) e para a verificação da normalidade da distribuição de frequência dos dados foi utilizado o teste de Kolmogorov-Smirnov, cujos valores de assimetria e curtose devem ser, respectivamente, próximos de zero e três para distribuições normais. O test $t$ de Student foi utilizado para comparar os teores de cobre e zinco nas duas profundidades dos solos sob vinhedos comerciais e experimentais. Também foi utilizada a análise de correlação linear simples de Pearson entre alguns atributos dos solos.

Para verificar a dependência espacial das variáveis, interpolar dados e elaborar os mapas, foi empregada a análise geoestatística segundo VIEIRA (2000). Foram construídos semivariogramas, partindo das pressuposições de estacionariedade da hipótese intrínseca e do cálculo da semivariância g(h) estimada pela equação 1 :

$$
\gamma(h)=\frac{1}{2 N(h)} \sum_{i=1}^{N}\left[Z\left(x_{i}\right)-Z\left(x_{i}+h\right)\right]^{2}
$$

em que $\mathrm{N}$ (h) é o número de pares dos valores medidos $\mathrm{Z}\left(\mathrm{x}_{\mathrm{i}}\right), \mathrm{Z}\left(\mathrm{x}_{\mathrm{i}+\mathrm{h}}\right)$, separados por um vetor $\mathrm{h}$. É esperado, segundo VieIRa (2000), que medições localizadas próximas sejam mais parecidas entre si do que aquelas separadas por grandes distâncias, isto é, que aumente $\gamma(\mathrm{h})$ com a distância $\mathrm{h}$ até um valor máximo, no qual se estabiliza em um patamar correspondente à distância limite de dependência espacial, sendo o alcance. Medições localizadas a distâncias maiores que o alcance terão distribuição aleatória, razão por que serão independentes entre si.

Os semivariogramas com dependência espacial foram ajustados com o modelo matemático de melhor correspondência. Os programas computacionais e procedimentos para construção e ajuste do modelo do semivariograma foram desenvolvidos por VIEIRA et al. (2002).

Calculou-se o grau de dependência espacial (GD), que é a proporção em porcentagem do "partial sill" $\left(C_{1}\right)$ em relação ao patamar $\left(C_{0}+C_{1}\right)$ (equação 2$)$, sendo, segundo Zimback (2001) e Trangmar et al. (1985), dependência fraca $<25 \%$, dependência moderada de $26 \%$ a $75 \%$ e dependência forte $>75 \%$.

$$
G D=\left(\frac{C_{1}}{C_{0}+C_{1}}\right) \cdot 100
$$

Uma vez que o semivariograma representa a variabilidade espacial dos dados, a análise geoestatística permitiu a análise dos dados, comparando-se os parâmetros de ajuste dos semivariogramas para cada uma das variáveis estudadas.
Havendo dependência espacial demonstrada pelo semivariograma, pode-se estimar valores para qualquer outro local que não foi amostrado, usando-se a krigagem, que segundo VIEIRA et al. (2002), estima os valores com condições de não-tendenciosidade e com desvios mínimos em relação aos valores conhecidos, ou seja, com variância mínima (Equação 3):

$$
\sum_{j=1}^{N} \lambda_{j} \gamma\left(X_{i}, X_{j}\right)+\mu=\gamma\left(X_{i}, X_{0}\right), i=1, N \sum_{j=1}^{N} \lambda_{j}=1
$$

Em que $\left(X_{i}, X_{j}\right)$ é a semivariância estimada, usando o modelo ajustado ao semivariograma, correspondente à distância entre os pontos localizados na posição $X_{i}$ e $X_{j}$ e $\gamma\left(X_{i}, X_{0}\right)$ é a semivariância correspondente à distância entre os pontos localizados na posição $X_{i}$ e $X_{0}$. Valores de peso 1 e um valor do multiplicador de Lagrange, $m$, associado com a minimização da variância, são gerados e com os valores de $1_{i}$ podem-se estimar valores $(Z)$ no espaço amostrado para qualquer posição $X_{0}$. Com os valores estimados (Equação 4), foram construídos mapas de isolinhas, em função da coordenada geográfica. O uso da krigagem como interpolador permite mostrar a variabilidade espacial de determinada área, pois a partir dela é possível a construção de mapas de isolinhas.

$$
z^{*}\left(x_{0}\right)=\sum_{i=1}^{n} \lambda_{i} Z\left(x_{i}\right)
$$

Para verificar a existência de correlação espacial entre o cobre e o zinco, foi realizada a análise de semivariograma cruzado ou "cross" semivariograma, que evidencia a ocorrência ou não da dependência espacial entre duas variáveis amostradas nos mesmos locais e que apresentam certo grau de correlação.

Se existir dependência espacial para cada uma das variáveis $\left(Z_{1}\right.$ e $\left.Z_{2}\right)$, a existência de dependência espacial entre $Z_{1}$ e $Z_{2}$ pode ser testada. $O$ cálculo do semivariograma cruzado é usado para avaliar a autocorrelação espacial entre os pares de variáveis $\left(Z_{1}\right.$ e $Z_{2}$ ) (Equação 5).

$$
\frac{1}{2 N(h)}(\mathrm{h})=\sum_{I=1}^{N}\left[Z_{1}\left(x_{i}\right)-Z_{1}\left(x_{i}+h\right)\right]\left[Z_{2}\left(x_{i}\right)-Z_{2}\left(x_{i}+h\right)\right]
$$

Segundo VAuclin et al. (1983), se existir dependência espacial para cada uma das variáveis (semivariogramas) e também entre elas ("cross" semivariograma) a cokrigagem pode ser usada para estimar valores da variável de menor densidade de amostragem (variável principal) em relação à densamente amostrada (variável secundária). Entretanto, como os pontos de amostragem do cobre e do zinco são exatamente os mesmos e na mesma 
densidade de amostragem, foi construído apenas o semivariograma cruzado para verificar a correlação da dependência espacial entre um e outro.

\section{RESULTADOS E DISCUSSÃO}

Pela estatística descritiva dos dados (Tabela 1), observa-se que os valores das médias e medianas (medidas de tendência central) são diferentes, e que a mediana e sempre é menor do que a média. As frequências foram maiores para baixos valores de cobre e zinco, correspondentes às áreas com usos diferentes de vinhedos. Os valores máximos observados foram em área atualmente utilizada com a cultura da pupunha, cultivada por várias décadas com vinhedos, demonstrando o alto poder de permanência do cobre nos solos.

As diferenças nos valores de cobre e zinco pelo uso do solo, proporcionaram anormalidade na distribuição de frequência para a maioria dos dados, com exceção do zinco na profundidade de 0,0 a 0,15 $\mathrm{m}$ com distribuição normal segundo o teste de Kolmogorov-Smirnov (Tabela 1). Em razão da nãonormalidade dos dados, assume-se que os desvios não são aleatórios e que a média não pode ser adotada como boa estimativa da tendência central dos valores da população, e nesse caso, de acordo com AMAro FILHO et al. (2007), torna-se necessário o uso da ferramenta auxiliar geoestatística, que define a distância amostral a partir da qual a independência espacial das amostras passa a ser satisfeita. Baixos valores de cobre e zinco em área de vinhedo e altos em área com pupunha, também foram responsáveis pelos altos coeficientes de variação, todos acima de 70\%, com base no critério de WARRICK e NIELSEN (1980) que classifica o coeficiente de variação como baixo < $12 \%$, médio de $12 \%$ a $60 \%$ e alto $>60 \%$. Os resultados de análises do solo costumam ser de valores altos e muito altos de coeficiente de variação (CARVALHO et al., 2003), especialmente no caso do presente trabalho que considera amostras de solos bastante heterogêneos quanto ao uso e coletadas a distâncias de dezenas de metros.

Considerando a totalidade das amostras na camada de 0,0 a $0,15 \mathrm{~m}$, os teores de cobre variaram de 0,6 a $18,5 \mathrm{mg} \mathrm{dm}^{-3}$, com média de $3,68 \mathrm{mg} \mathrm{dm}^{-3}$; e na camada de 0,15 a $0,30 \mathrm{~m}$ os teores variaram de 0,4 a $15,5 \mathrm{mg} \mathrm{dm}^{-3}$, com média de $2,98 \mathrm{mg} \mathrm{dm}^{-3}$. Para a totalidade das amostras, os teores de zinco na camada de 0,0 a $0,15 \mathrm{~m}$ variaram de 0,6 a $41,6 \mathrm{mg} \mathrm{dm}^{-3}$, com média de 7,16 $\mathrm{mg} \mathrm{dm}^{-3} \mathrm{e}$, na camada de 0,15 a $0,30 \mathrm{~m}$ os teores variaram de 0,3 a $261,7 \mathrm{mg} \mathrm{dm}^{-3}$, com média de 4,43 $\mathrm{mg} \mathrm{dm}^{-3}$. Os valores discrepantes, como o de $261,7 \mathrm{mg} \mathrm{dm}^{-3}$ de zinco de 0,15 a $0,30 \mathrm{~m}$ em uma área de vinhedo comercial foram eliminados das análises estatísticas listadas na tabela 1, por serem muito superior aos demais valores, e não representarem as tendências neste uso do solo.

Nas áreas com vinhedos comerciais, os teores de cobre na camada de 0,0 a 0,15 m variaram de 9,6 a $15,5 \mathrm{mg} \mathrm{dm}^{-3}$, com média de $12,1 \mathrm{mg} \mathrm{dm}^{-3}$ e nos experimentais variaram de 2,4 a $10,0 \mathrm{mg} \mathrm{dm}^{-3}$, com média de $5,5 \mathrm{mg} \mathrm{dm}^{-3}$. Na camada de 0,15 a $0,30 \mathrm{~m}$, os teores nos vinhedos comerciais variaram de 8,0 a $14,6 \mathrm{mg} \mathrm{dm}^{-}$ 3 , com média de $10,7 \mathrm{mg} \mathrm{dm}^{-3}$ e nos experimentais variaram de 1,8 a $4,9 \mathrm{mg} \mathrm{dm}^{-3}$, com média de $3,0 \mathrm{mg} \mathrm{dm}^{-}$ ${ }^{3}$. Com base no teste $t$ de Student também houve diferença significativa entre os teores de cobre nos vinhedos comerciais e experimentais em nível de $0,1 \%$ de probabilidade, para as duas camadas consideradas.

Resultados semelhantes aos de cobre nas áreas de vinhedos comerciais e experimentais, também foram verificados para zinco. Na camada de 0,0 a 0,15 $\mathrm{m}$ os teores do elemento nos vinhedos comerciais variaram de 6,7 a $25,0 \mathrm{mg} \mathrm{dm}^{-3}$, com média de 16,5 $\mathrm{mg} \mathrm{dm}^{-3}$ e nos experimentais variaram de 3,2 a 24,6 $\mathrm{mg} \mathrm{dm}^{-3}$, com média de $10,7 \mathrm{mg} \mathrm{dm}^{-3}$; e na camada de 0,15 a $0,30 \mathrm{~m}$ os teores nos vinhedos comerciais variaram de 7,2 a $21,4 \mathrm{mg} \mathrm{dm}^{-3}$, com média de 14,4 $\mathrm{mg} \mathrm{dm}^{-3}$ e nos experimentais variaram de 1,6 a 15,4 $\mathrm{mg} \mathrm{dm}{ }^{-3}$, com média de $4,8 \mathrm{mg} \mathrm{dm}^{-3}$.

Tabela 1. Resultado da estatística descritiva para os teores de cobre e zinco no solo, extraídos com DTPA, em mg dm ${ }^{-3}$ em duas profundidades de coleta $(0,0$ a $0,15 \mathrm{~m}$ e 0,15 a 0,30 m) e o teste de normalidade de Kolmorogov Smirnov

\begin{tabular}{lccccccc}
\hline Profundidade & Média & Mediana & Variância & CV $(\%)$ & Assimetria & Curtose & Teste KS* D \\
\cline { 2 - 6 } m & 3,68 & 2,5 & 8,77 & 80,37 & 1,74 & 3,00 & 0,230 (não normal) \\
$0,0-0,15$ & 2,98 & 2,2 & 7,60 & 92,62 & 2,52 & 6,53 & 0,240 (não normal) \\
$0,15-0,30$ & & & & & & \\
Zinco & 7,16 & 6,1 & 26,98 & 72,59 & 1,51 & 2,10 & 0,128 (normal) \\
$0,0-0,15$ & 4,43 & 3,3 & 16,45 & 91,56 & 2,05 & 5,03 & 0,170 (não normal) \\
$0,15-0,30$ & & & & & &
\end{tabular}

* KS (Kolmogorov Smirnov), D tab $(0,05)=0,138$. 
Com base no teste $t$ de Student houve diferença significativa entre os teores de zinco nos vinhedos comerciais e experimentais em nível de 5\% de probabilidade, para as duas profundidades consideradas. Os teores de cobre nos solos sob vinhedos proporcionaram correlação positiva com os teores de zinco $(r=0,40)$, matéria orgânica $(\mathrm{r}=0,54), \mathrm{pH}(\mathrm{r}=0,33)$ e saturação por bases $(r=0,42)$, com coeficientes de correlação significativos em nível de $2 \%$ de probabilidade, indicando que quanto maiores os valores desses atributos, maiores tendem a ser os teores de cobre no solo. Com teores de zinco somente houve correlação com os teores de cobre. A geodisponibilidade do cobre é fortemente influenciada pelo teor da matéria orgânica e tipos de substâncias húmicas presentes no solo (Wu et al., 2002). Elevados teores de cobre podem diminuir a atividade microbiológica, e assim, a taxa de decomposição da matéria orgânica no solo (PARAT et al., 2002). Desta forma, é possível inferir, de acordo com os resultados verificados, que esses aspectos possam estar sendo alterados na área de estudo, em razão do altos teores de cobre observados. Esse fato torna-se mais agravante nas áreas com vinhedo comercial, em consequência dos maiores teores desse elemento constatados no solo.

Apesar das grandes distâncias entre as amostras, foi observada dependência espacial, sendo ajustados os semivariogramas (Figura 2) com patamares bem definidos e razão de dependência espacial (GD) forte para o cobre e moderada para o zinco. Os semivariogramas foram ajustados pelo modelo Gaussiano, pois foi o que melhor definiu o efeito pepita, com altos valores de coeficientes de correlação. O efeito pepita foi menor, mais próximo de zero, para os teores de cobre (Figuras 2a e 2b), e maior para os teores de zinco, ou seja, mais elevado, resultando em menor grau de dependência espacial (Figuras 2c e 2d). $\mathrm{O}$ alcance variou de $650 \mathrm{~m}$ para zinco $(0,15$ a $0,30 \mathrm{~m})$ a $950 \mathrm{~m}$ para cobre $(0,15$ a $0,30 \mathrm{~m})$.

Houve semelhança no comportamento espacial dos teores de cobre e zinco nas duas profundidades (Figura 3 a, b, c e d), indicando que o aumento da concentração dos elementos está ocorrendo nas duas camadas. Os teores de cobre e zinco mais elevados coincidem com as áreas sob vinhedos (Figura 3e), principalmente na profundidade de 0,0 a $0,15 \mathrm{~m}$. Em virtude desta semelhança verificou-se a correlação espacial entre o cobre e o zinco nas duas profundidades por meio da construção do semivariograma cruzado do cobre como variável principal e zinco como secundária (Figuras 4a e 4b). Houve correlação espacial positiva e dependência espacial para a correlação entre o cobre e o zinco na profundidade de 0,15 a $0,30 \mathrm{~m}$ (Figura $4 \mathrm{~b}$ ), com ajuste gaussiano, $\mathrm{r}^{2}=0,80$ e alcance $759 \mathrm{~m}$, visto que não foi possível ajustar o semivariograma cruzado entre cobre e zinco na camada de 0,0 a $0,15 \mathrm{~m}$ como mostra a figura 4a. O grau de dependência espacial também foi maior na profundidade de 0,15 a $0,30 \mathrm{~m}$ tanto para o cobre quanto para o zinco (Tabela 2) o que favoreceu a ocorrência da dependência espacial entre os elementos correlacionados nesta profundidade. Segundo OrTIZ et al. (2007), a elaboração do semivariograma cruzado é uma fase crítica do processo por ser muito exigente, pois requer ajuste dos semivariogramas diretos e também do semivariograma cruzado com boa representação da variabilidade espacial através da correlação espacial de duas variáveis. No semivariograma cruzado, obtido por ORTIz et al. (2007), para argila no horizonte superficial e argila no horizonte subsequente, observou-se a mesma estrutura básica dos semivariogramas diretos e todos os modelos foram ajustados por funções exponenciais. Desta forma, a obtenção dos mesmos modelos de ajustes e estrutura dos semivariogramas diretos de Cu e Zn e do semivarigrama cruzado entre as duas vem reforçar a forte dependência espacial entre elas na profundidade de $0,15 \mathrm{a} 0,30 \mathrm{~m}$.
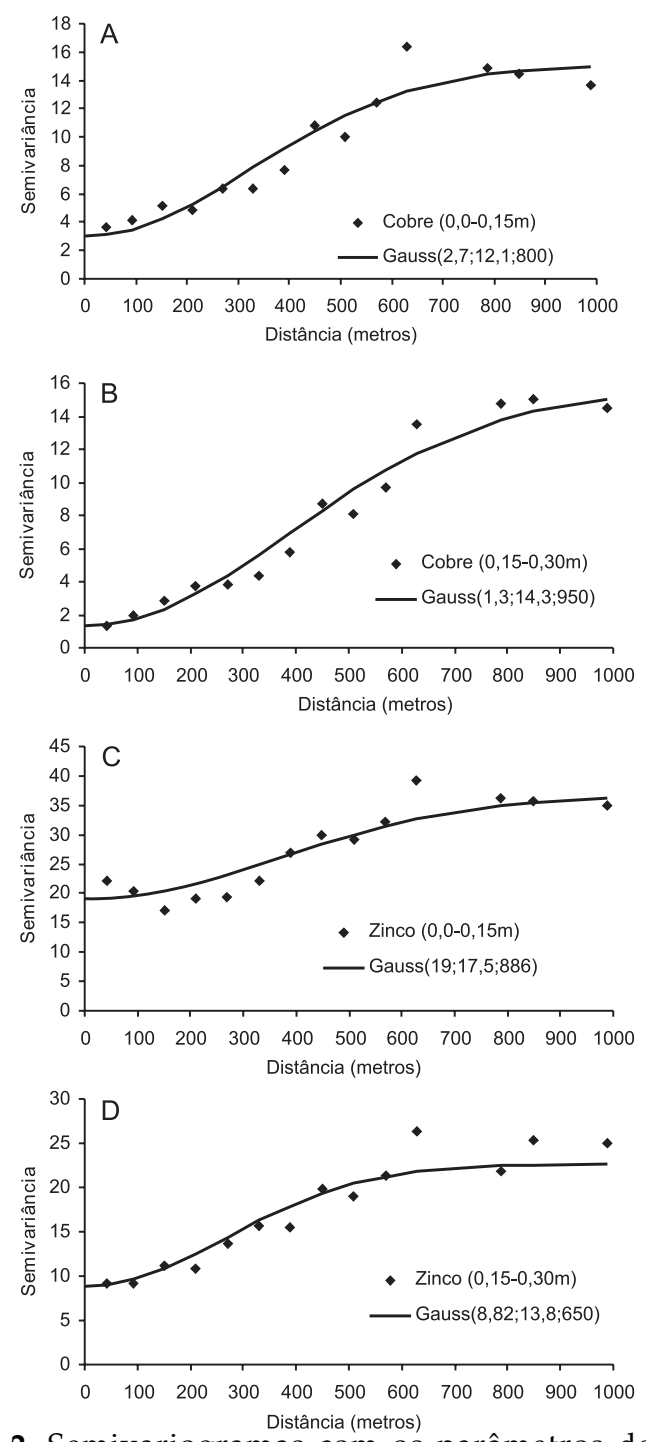

Figura 2. Semivariogramas com os parâmetros de ajuste Gaussiano para o teor de cobre, (a) 0,0 a 0,15 m, (b) 0,15 a $0,30 \mathrm{~m}$, e zinco (c) 0,0 a $0,15 \mathrm{~m}$, (d) 0,15 a $0,30 \mathrm{~m}$. Modelo (Co; $\mathrm{C} 1 ; \mathrm{a})$. 


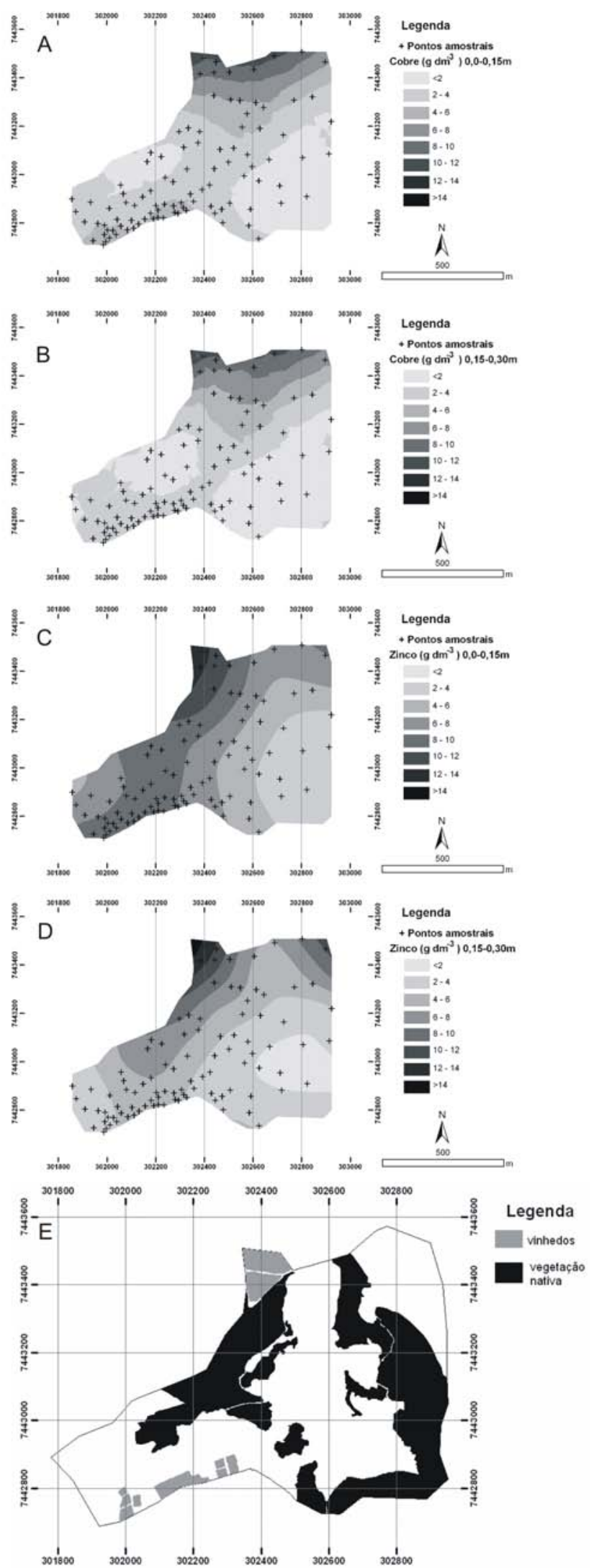

Figura 3. Distribuição espacial dos teores de cobre (a) 0,0 a $0,15 \mathrm{~m}$; (b) 0,15 a $0,30 \mathrm{~m}$; zinco, (c) 0,0 a $0,15 \mathrm{~m}$, (d) 0,15 a $0,30 \mathrm{~m}$ nos solos estimados por krigagem e (e) as áreas com vinhedos e vegetação nativa.

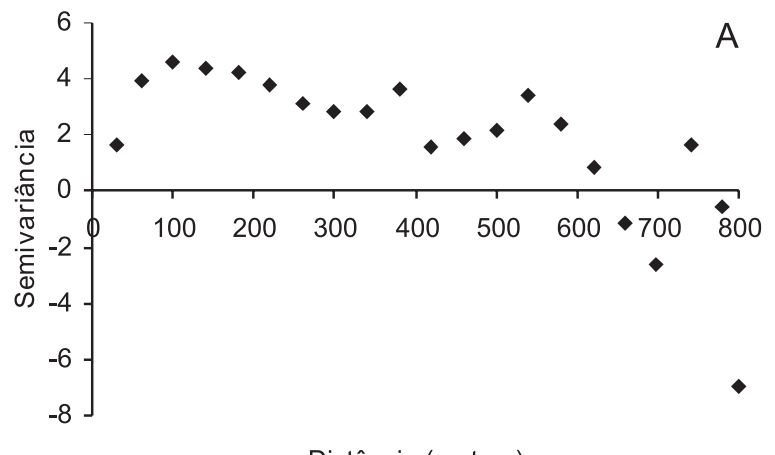

$\mathrm{Cu} \times \mathrm{Zn}(0,0-0,15 \mathrm{~m})$

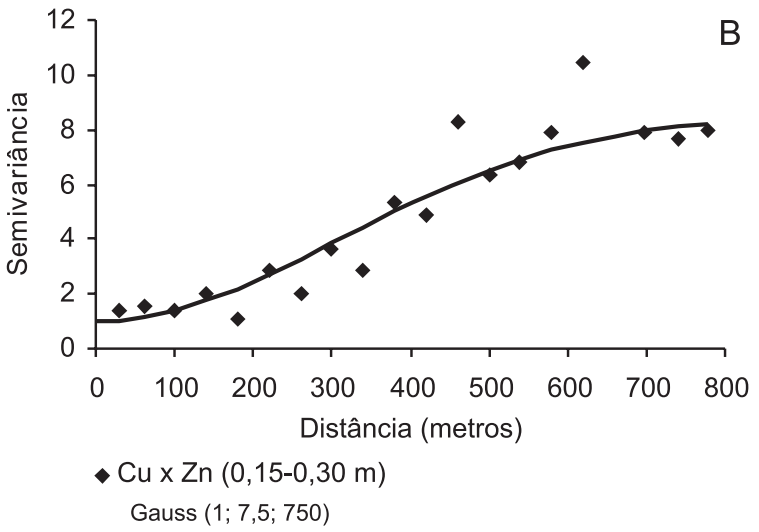

Figura 4. Semivariogramas cruzados entre cobre e zinco, (a) 0,0 a $0,15 \mathrm{~m}$ e (b) 0,15 a 0,30 m. Modelo (Co; C1; a).

Quando existe correlação cruzada entre duas variáveis, como ocorreu neste caso do cobre e do zinco, a análise de correlação espacial por meio do semivariograma cruzado é válida. O passo seguinte seria a interpolação por cokrigagem, com a construção do mapa da variável primária a partir da correlação espacial encontrada com a secundária, mas no presente caso, onde a densidade amostral do cobre e do zinco são iguais, não haverá ganho no uso de cokrigagem em relação à krigagem simples. Segundo Goovaerts (1997), as estimativas de krigagem e cokrigagem são essencialmente as mesmas para o caso isotrópico e as diferenças entre as estimativas aumentam quando os dados secundários são mais numerosos que os dados primários.

VieIra (2000) mostrou que a dependência espacial do nitrogênio expressa pelo semivariograma foi tão forte que mascarou o efeito do carbono tanto no semivariograma cruzado carbono versus nitrogênio como também no mapa obtido por cokrigagem e concluiu, ser supérfluo e desnecessário usar a cokrigagem.

Comparando-se os teores de cobre dos solos sob vinhedos com aqueles sob vegetação nativa (Figura 3e), na camada de 0,0 a $0,15 \mathrm{~m}$, observa-se que nos vinhedos a média foi de $6,7 \mathrm{mg} \mathrm{dm}^{-3}$ e na vegetação nativa, de 1,9 $\mathrm{mg} \mathrm{dm}{ }^{-3}$; na camada de 0,15 a $0,30 \mathrm{~m}$ a média dos 
Tabela 2. Parâmetros do modelo Gaussiano ajustado aos semivariogramas: efeito pepita $\left(\mathrm{C}_{0}\right)$, variância estrutural $\left(C_{1}\right)$, alcance $(a)$, razão de dependência espacial (GD) e coeficiente de correlação para os teores de cobre e zinco, extraídos com DTPA, em duas profundidades (0,0 a $0,15 \mathrm{~m}$ e 0,15 a $0,30 \mathrm{~m})$.

\begin{tabular}{|c|c|c|c|c|c|}
\hline Profundidade & $\mathrm{C}_{0}$ & $\mathrm{C}_{1}$ & $\mathrm{a}(\mathrm{m})$ & GD & Coeficiente correlação \\
\hline$\overline{\mathrm{m}}$ & & & & $\%$ & $\mathrm{r}^{2}$ \\
\hline \multicolumn{6}{|l|}{ Cobre } \\
\hline $0,0-0,15$ & 2,7 & 12,10 & 800 & 80,13 & 0,88 \\
\hline $0,15-0,30$ & 1,3 & 14,3 & 950 & 91,67 & 0,96 \\
\hline \multicolumn{6}{|l|}{ Zinco } \\
\hline $0,0-0,15$ & 19,00 & 17,5 & 886 & 47,95 & 0,86 \\
\hline $0,15-0,30$ & 8,82 & 13,8 & 650 & 61,01 & 0,90 \\
\hline
\end{tabular}

vinhedos foi de $4,4 \mathrm{mg} \mathrm{dm}^{-3}$ e na vegetação nativa, de 2,1 $\mathrm{mg} \mathrm{dm}^{-3}$. Com base no teste $\mathrm{t}$, de Student, houve diferença significativa entre os teores de cobre nos vinhedos e na vegetação nativa a 5\% de probabilidade. Resultados semelhantes foram observados para o zinco para a camada de 0,0 a $0,15 \mathrm{~m}$, em que a média nos vinhedos foi de $11,7 \mathrm{mg} \mathrm{dm}^{-3}$ e na vegetação nativa 7,8 $\mathrm{mg} \mathrm{dm}{ }^{-3}$, com diferença significativa em nível de $10 \%$ segundo o teste $t$. Na camada de 0,15 a 0,30 m os teores de zinco não tiveram diferença estatística significativa com teores médios de 6,3 e 6,4 $\mathrm{mg} \mathrm{dm}^{-3}$, respectivamente, para solos de vinhedos e vegetação nativa. Desta forma, os resultados revelam, em geral, que o uso do solo alterou de maneira significativa os teores de $\mathrm{Cu}$ e $\mathrm{Zn}$, evidenciando o potencial em aumentar os teores destes elementos no solo com o uso de vinhedos.

\section{CONCLUSÕES}

1. O uso do solo com vinhedos aumenta os teores de Cu e Zn disponíveis, possivelmente em razão de aplicação de agroquímicos no manejo fitossanitário.

2. Verificam-se correlações positivas significativas dos teores de cobre no solo com os de zinco, pH, matéria orgânica e saturação por bases, mostrando a forte relação entre estes atributos do solo.

3. Com a análise geoestatística utilizando semivariograma, notam-se nos elementos $\mathrm{Cu}$ e $\mathrm{Zn}$ forte estrutura de distribuição espacial, o que permitiu a obtenção de mapas de isolinhas de forma confiável e a partir dos quais se observaram as tendências de aumento dos teores desses dois elementos conforme o uso do solo, especificamente com maiores teores nas áreas com vinhedos e menores teores nas áreas com mata nativa.

4. A análise geoestatística utilizando o semivariograma cruzado revela haver forte correlação espacial positiva entre os teores de $\mathrm{Cu}$ e $\mathrm{Zn}$ na profundidade de 0,15 a0,30 m, apesar da grande distância entre os pontos amostrais e a diversidade de usos da terra da área estudada.

\section{AGRADECIMENTOS}

Aos pesquisadores José Luiz Hernandes, Jener Fernando Leite de Moraes e Mário José Pedro Júnior pelas colaborações prestadas, facilitando a realização deste trabalho. À FAPESP, processo 04/03847-5, pelo financiamento.

\section{REFERÊNCIAS}

AMARO FILHO, J.; NEGREIROS, R.F.D; ASSIS JÚNIOR, R.N.; MOTA, J.C.A. amostragem e variabilidade espacial de atributos físicos de um Latossolo Vermelho em Mossoró, RN. Revista Brasileira de Ciência do Solo, 31, 3, p.415-422, 2007.

ALLEONI, L.R.F.; NACHTIGALL, G.R.; CAMBRI, M.A.; NOGUEIROL, R.C.; LOPES, C.M. Disponibilidade de cobre em solos de vinhedos após aplicação de calda bordaleza por vários anos. In: CONGRESSO BRASILEIRO DE CIÊNCIA DO SOLO, 29., 2003, Ribeirão Preto. Anais... Viçosa: Sociedade Brasileira de Ciência do Solo, 2003, CD-ROM.

BRUN, L.A.; MAILLET, J.; RICHARTE, J.; HERRMANN, P.; REMY, J.C. Relationships between extractable copper, soil properties and copper uptake by wild plants in vineyard soils. Environmental Pollution, v.102, p.151-161, 1998.

CÂMARA, G.; MEDEIROS J.S. Geoprocessamento para projetos ambientais. São José dos Campos: INPE, 1996. (CD-ROM)

CAMARGO, O.A.; BORBA, R.P.; ALLEONI, L.R.F. Metais pesados: da cosmogênese aos solos brasileiros. In: CONGRESSO BRASILEIRO DE CIÊNCIA DO SOLO, 29., 2003, Ribeirão Preto. Anais... Viçosa: Sociedade Brasileira de Ciência do Solo, 2003. (CD-ROM)

CARVALHO, M.P.; TAKEDA, E.Y.; FREDDI, O.S. Variabilidade espacial de atributos de um solo sob videira em Vitório Brasil 
(SP). Revista Brasileira de Ciência do Solo, v.27, p.695-703, 2003.

CHAIGNON, V.; HINSINGER, P. A.Biotest for Evaluating Copper Bioavailability to Plants in a Contaminated Soil. Environmental Quality, v.32, p.824-833, 2003.

CHAIGNON, V.; SANCHEZ-NEIRA, I.; HERMANN, P.; JAILLARD, B.; HINSINGER, P. Copper bioavailability and extractability as related to chemical properties of contaminated soils from a vine-growing area. Environmental Pollution, v.123, p.229-238, 2003.

COSTA, M.C.R. Avaliação da contaminação ambiental por metais pesados em áreas rurais próximas a uma indústria de reciclagem de chumbo no vale do rio Paraíba do Sul - SP. 2002. 77f. Tese (Doutorado em Agronomia). Universidade Federal Rural do Rio de Janeiro-UFRRJ, Seropédica.

COUTO, E. G.; KLAMT, E. Variabilidade espacial de micronutrientes em solo sob pivô central no sul do Estado de Mato Grosso. Pesquisa Agropecuária Brasileira, v.34, p.23212329, 1999.

DELUISA, A.; GIANDON, P.; AICHNER, M.; BOTLAMI, P.; BRUNA, L.; LUPETTI, A.; NARDELLI, F.; STRINGARI, G. Copper pollution in Italian vineyard soils. Communication in Soil Science and Plant Analysis, v.27, p.1537-1548, 1996.

FACCHINELLI, A.; SACCHI, E.; MALLEN, L. Multivariate statistical and GIS-based approach to identify heavy metal sources in soils. Environmental Pollution, v.114, p.313324, 2001.

FADIGAS, F. S. Estimativas das concentrações naturais (pseudototal) de $\mathrm{Cd}, \mathrm{Co}, \mathrm{Cr}, \mathrm{Cu}, \mathrm{Ni}$, $\mathrm{Pb}$ e $\mathrm{Zn}$ em solos brasileiros e proposição de valores de referência utilizando técnicas da estatística multivariada. 2002. 116f. Tese (Doutorado em Agronomia). Universidade Federal Rural do Rio de JaneiroUFRRJ, Seropédica.

FELIX, F.F. Comportamento do cobre aplicado no solo por calda bordalesa. 2005. 85f. Dissertação (Mestrado em Agronomia). ESALQ, Universidade de São Paulo-USP, Piracicaba.

GAW, S.K.; WILKINS, A.L.; KIM, N.D; PALMER, G.T.; ROBINSON, P. Trace element and DDT concentrations in horticultural soils from the Tasman, Waikato and Auckland regions of New Zealand. Science of the Total Environment, v.355, p.31-47, 2006

GIOVANNINI, E. Toxidez por cobre em vinhedos. Pesquisa Agropecuária Gaúcha, v.3, p.115-117, 1997.

GOOVAERTS, P. Geostatistics for natural resources evaluation. New York: Oxford University Press, 1997. 476p.

NICHOLSON, F.A.; SMITH, S.R.; ALLWAY, B.J.; CARLTONSMITH, C.; CHAMBERS, B.J. An inventory of heavy metals inputs to agricultural soils in England and Wales. The Science of the Total Environment, v.311, p.205-219, 2003.
ORTIZ, J.O., FELGUEIRAS, C.A., DRUCK, S., MONTEIRO, A.M.V. Avaliação de procedimentos geoestatísticos de cokrigagem para determinação da distribuição espacial de propriedades de solos. In: MEIRELLES, M.S.P. CAMARA, G., ALMEIDA, C.M. (Ed). Geomática: modelos e aplicações ambientais. Brasília: Embrapa Informação tecnológica, 2007. p.387-408.

PAOLETTI, M.G.; SOMMAGIO, D.; FAVRETTO, M.R.; PETRUZZELLI, G.; PEZZAROSSA, B.; BARBAFIERI, M. Earthworms as useful bioindicators of agroecosystem sustainability in orchards and vineyards with different inputs. Applied Soil Ecology, v.10, p.137-150, 1998.

PARAT, C.; CHAUSSOD, R.; LÉVÊQUE, J.; DOUSSET, S.; ANDREUX, F. The relationship between copper accumulated in vineyard calcareous soils and soil organic matter and iron. European Journal of Soil Science, v.53, p.663-669, 2002.

RAIJ, B. van ; ANDRADE, J.C.; CANTARELLA, H.; QUAGGIO, J.A. Análise química para avaliação da fertilidade de solos tropicais. Campinas: Instituto Agronômico, 2001. v. 1. 285 p.

RAMALHO, J.F.G.P.; AMARAL SOBRINHO, N.M.B.; VELLOSO, A.C.X. Contaminação da microbacia de Caetés com metais pesados pelo uso de agroquímicos. Pesquisa Agropecuária Brasileira, v.35, p.1289-1303, 2000.

RAMOS, M.C. Metals in vineyard soils of the Penedès area (NE Spain) after compost application. Journal of Environmental Management, v.78, p.209-215, 2006.

RAMOS, M.C.; LÓPEZ-ACEVEDO, M. Zinc levels in vineyard soils from the Alt Penedès-Anoia region (NE Spain) after compost application. Advances in Environmental Research, v.8, p.687-696, 2004.

SRIVASTAVA, R.M. Describing spatial variability using geostatistics analysis. In: SRIVASTAVA, R.M.; ROUHANI, S.; CROMER, M.V.; et al. (Ed). Geostatistics for environmental and geotechnical applications. West Conshohocken: American Society for Testing and Materials, 1996. p.13-19.

STAR, J.; ESTES, J. Geographic information systems: an introduction. Englewood Cliffs: Prentice Hall, 1990. 665p.

TRANGMAR, B.B.; YOST, R.S.; UEHARA, G. Applications of geostatistics to spatial studies of soil properties. Advances in Agronomy, v.38, p.45-94, 1985.

VAUCLIN, M., VIEIRA, S.R., VAUCHAUD, G., NIELSEN, D.R.. The use of cokriging with limited field soil observation. Soil Science Society of America Journal, v.47, p.175-84, 1983.

VIEIRA, S.R. Uso de geoestatística em estudos de variabilidade espacial de propriedades do solo. In: NOVAIS, R. F. (Ed.). Tópicos em Ciência do Solo 1. Viçosa: Sociedade Brasileira de Ciência do Solo, 2000. p.3-87. 
VIEIRA, S.R.; MILLETE, J.A.; TOPP, G. C.; REYNOLDS, W.D. Handbook for Geostatistical analysis of variability in soil and meteorological parameters. In: ALVAREZ, V. H. (Ed.). Tópicos em Ciência do Solo 2. Viçosa: Sociedade Brasileira de Ciência do Solo, 2002. p 1-45.

VIEIRA, S.R. Variabilidade espacial de argila, silte e atributos químicos em uma parcela experimental de um Latossolo Roxo de Campinas (SP). Bragantia, v.56, p.181-190, 1997.

WARRICK, A.W.; NIELSEN, D.R. Spatial variability of soil physical properties in the field. In: HILLEL, D.,(Ed.) Applications of soil physics. New York, Academic Press, 1980. p.319-344.

WEBSTER, R. 1985. Quantitative spatial analysis of soil in the field. Advance in Soil Science, v.3, p.1-70, 1985.

WU, J; WEST, L.J.; STEWART, D.I. Effect of humic substances on $\mathrm{Cu}(\mathrm{II})$ solubility in kaolin-sand soil. Journal of Hazardous Materials, v.94, p.223-238, 2002.

ZIMBACK, C.R.L. Análise especial de atributos químicos de solo para o mapeamento da fertilidade do solo. 2001, 114p. Tese de livre docência - Faculdade de Ciências Agronômicas/ Unesp, Botucatu. 